\title{
The Design of Smart Battery Management Systems
}

\author{
Peide Liu \\ Information Management School, Shandong Economic University. Jinan 250014, P.R.China \\ Email: Peide.liu@gmail.com \\ Xiujuan Zhang * \\ Information Management School, Shandong Economic University. Jinan 250014, P.R.China \\ Email: zhangxiujuan68@163.com
}

\begin{abstract}
Aiming to the defect of expandability and reliability of Li-Ion battery of protection circuit, the multisection series connection Li-ion battery management system was designed based on X3100 chip. The hardware and software design of the system was elaborated. The system can realize the measure, management and auto-protection for various parameters and complete the calculation for tens of command parameters and realize the data exchange with HOST by SMBus. Applications show that the system is characterized by simple structure, perfect function and stable and reliable operation and can be used in the smart battery model of laptop computer, Electric bicycle and other portable equipments.
\end{abstract}

Index Terms-Smart Battery; SMBus; management system

\section{INTRODUCTION}

Smart Battery is to realize the intelligence of battery on the base of common chemical battery and smart battery management system (or called communication control protection system). Smart Battery can monitor the state of battery and communicate directly with smart charger and tell the charge requirement to smart charger at any time. Smart Battery also can communicate directly with smart equipment (such as laptop computer mainframe) through SMBus. Using Smart Battery can indicate and monitor accurately the state of battery and prolong the life of battery. The key technology of Smart Battery is Smart Battery management system.

Following the popularization and development of portable information products, the demand for Smart Battery is getting greater and greater, especially the development of laptop computer, Smart Battery has become its standard configure. At present, the first application field of Smart Battery is the battery of laptop computer; the second is various portable electronic products such as Video Camera/Recorder, portable DVD, digital camera, mobile phone. After being improved properly, it can be applied on the control and protection of power battery and also the electric bicycle, electric tool and electric Motorbike. So it has wide application fields and market prospect [1-3].

Smart Battery was proposed originally by Intel and Duracell in 1995. But it was not developed very well for lack of corresponding control chip and the support of BIOS in laptop computer. Until 1998, following the advent of Microsoft Windows 98 operation system which supports OnNow power management function and ACPI interface, it began to develop and became the real industry standard. At that time, Smart Battery only provided some simple functions such as basic electrical measurement and the editions of Smart Battery Data format protocol and System Manager Bus protocol are all 1.0 edition. The Smart Battery Data format protocol was upgraded to 1.1A on August, 1999. System Manager Bus protocol was upgraded to 2.0 edition on August, 2000. At present, the main management systems are as follows:

(1) Voltage protection scheme: The scheme belongs to the type of hardware protection. In early period, to solve the problem of over voltage and over discharge, using separate component to realize the protection for battery voltage and realizing the protection for charge maximum voltage and discharge minimum voltage by circuit; and then simple integrated circuit was used to realize the similar function. The scheme is simple to realize. But it only realizes the basic security protection and lacks the smart protection and control.

(2) Voltage and temperature protection scheme: The scheme belongs to the type of hardware protection. Separate component or simple integrated circuit was used to realize the protection of voltage and temperature. And it can basically satisfy the request of battery security, but it also lack the smart protection and control.

(3) Simple MCU control scheme[4]: Simple MCU is used to control the protection of circuit and complete the simple management. The scheme has higher reliability than the former two schemes and it improves the life of the battery and realizes partly intelligentization.

Aiming to the defect of current Li-ion battery management system, a low cost smart management system was designed. The system can realize the measure, management and auto-protection for various parameters.

Corresponding author :Xiujuan Zhang 


\section{The Principle OF SMART Li-ION BATTERY MANAGEMENT SYSTEM ARCHITECTURE}

The structure and composition of Smart Battery management system is shown in following Fig.1. The system includes Host, Manager, Charger, Battery, and Switcher, etc. They provide SMBus communication, system state, data and control with each other.

\section{A System Host(SBH)}

It is the main part of laptop computer or other portable equipments. It provides bus to get the power supply status and battery data, and it is applied on the application management and state display of power.

\section{B. System Manager(SBSM)[5][6]}

It is the part of system management. It controls the selection of DC/AC, the selection among cells system and the work of charge part. It exchanges data and state with Battery.Iindex System Establishment System Host(SBH).

\section{Smart Charger(SBC)[7]}

It is the part of smart charge management. It provides the charge current of CC-CV according to the data of Battery; it adjusts its own charge way according to the state of battery broadcasting

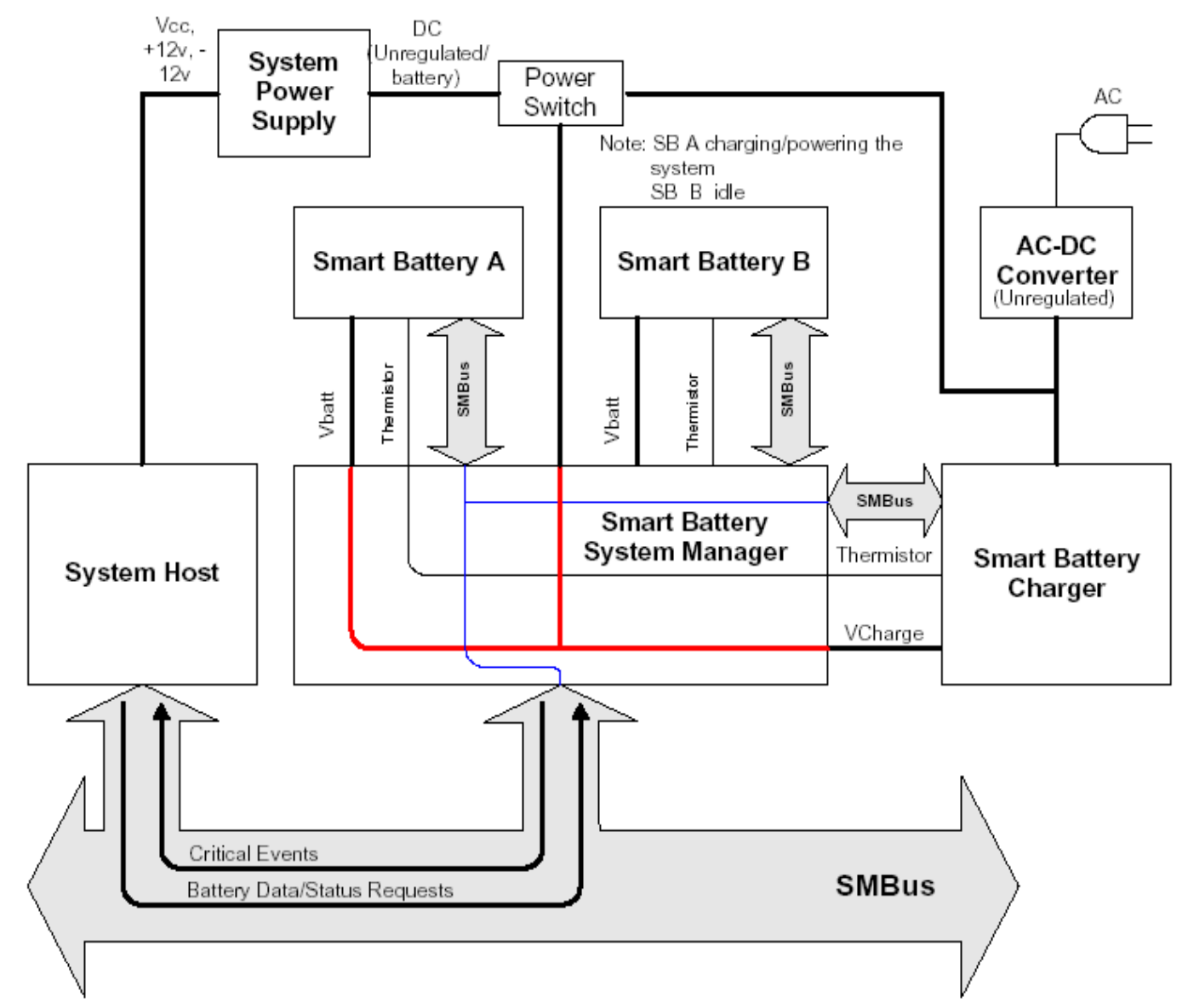

Fig.1 The structure and composition diagram of Smart Battery management system

\section{Smart Battery}

It is a unit composed of Battery and control part. For system, it is like a peripheral device. It completes automatically the functions such as parameters measure, calculation, management and protection for Battery. It is the system which was researched by our project.

\section{E. System Management Bus (SMBus) [8]}

SMBus is a bus that is used to communicate among the devices of Smart Battery system. Each device has a address by which the data exchange among various devices can be realized. The structure diagram is shown in Fig.2.

\section{The Hardware Design of MANAgement System}

\section{A. The design plan of hardware circuit}

In aspect of hardware, x3100/1 of American Xicor Company was selected as the Battery control chip [9]. In the aspect of MCU, Considering the request of measure accuracy, the accuracy of AD converter must reach over 10 Bit. Considering the request of capacity and function 
of program, we chose firstly the series as GC1318. The principle diagram of hardware is shown in Fig.3.

The system mainly includes the following parts: MCU, Battery, measure and protection, balance circuit, SMBus, FET and LED indication.

The measure and protection chip x3100 has power circuit, which provides power for the whole system. The chip can check automatically battery voltage and check current under the control of MCU and measure the voltage of resistance and then transfer it to MCU. X3100 can protect automatically the voltage, current and short circuit according to the parameters. X3100 connects the control balance circuit with FET circuit, and controls the actions of the two circuits under the order of MCU. MCU is the central-control part of the system; it measures the voltage and current by gating control for x3100; it controls balance circuit and FET circuit by SPI communication; it implements the calculation of electric capacity, the calculation of sbdata parameters, charge/discharge control, action control, balance action control and SMBus communication according to the parameters measured; it controls various functions such as temperature measure and electric capacity indication; it also implements the self-study of electric capacity and the calculation of period. In a word, it makes sure that the system has an accurate, stable and security work.

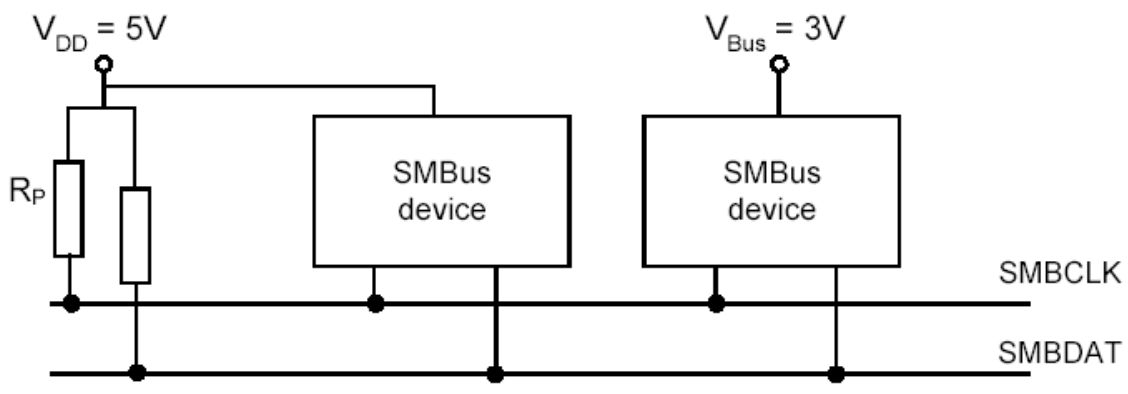

Fig. 2 The structure diagram of System Management Bus

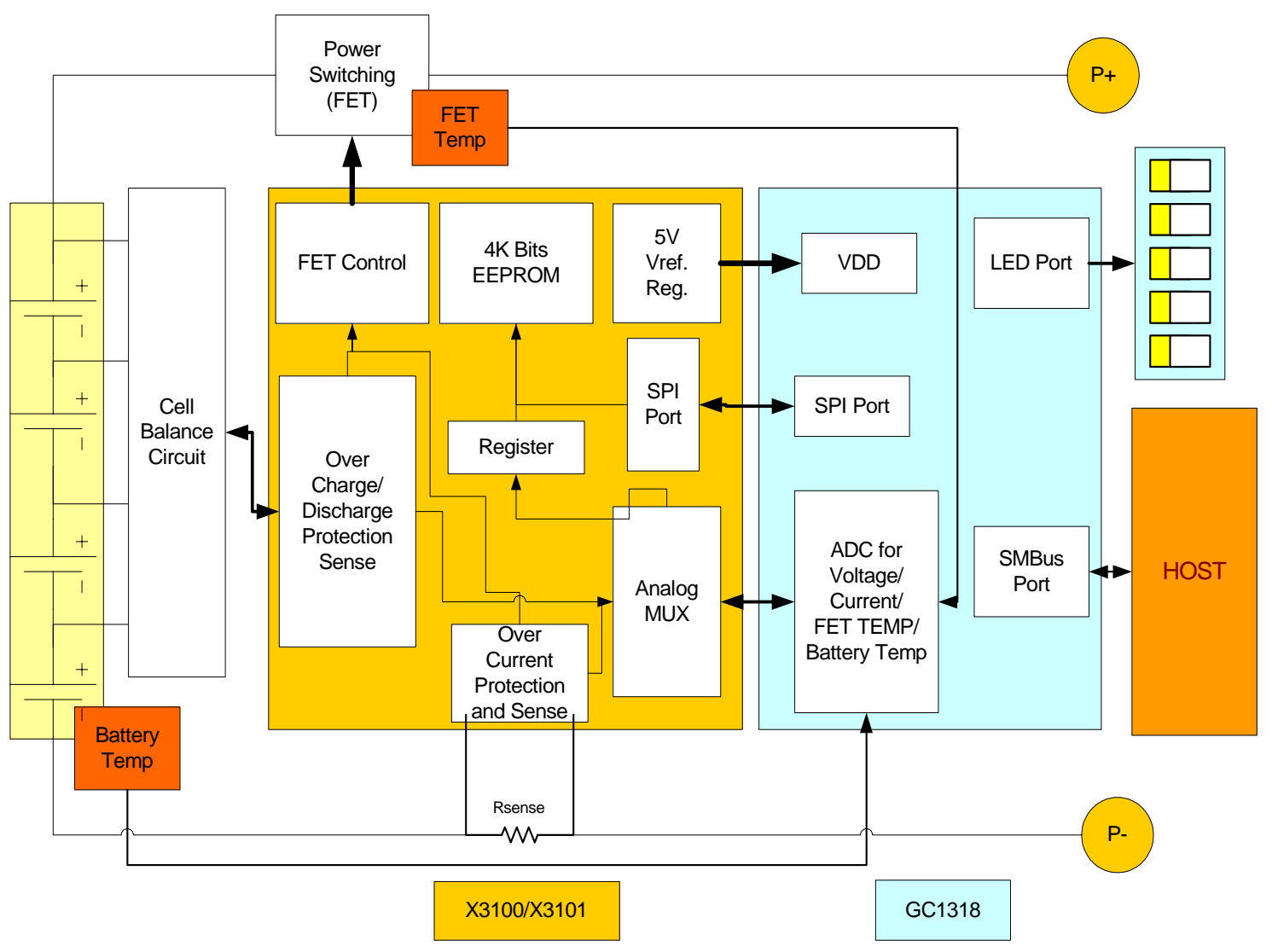

Fig.3 The principle diagram of Smart Battery hardware 


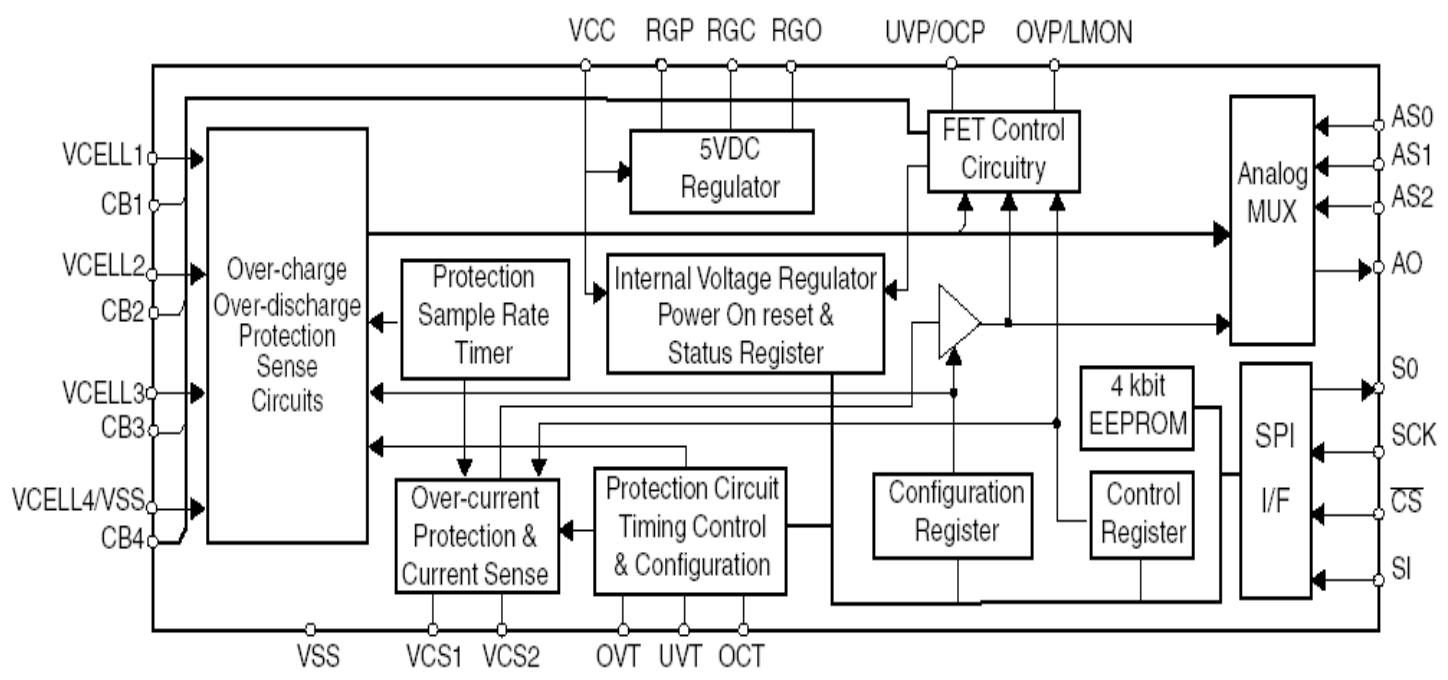

Fig.4 the structure diagram of x3100

\section{B. X3100 Circuit}

X3100 proposed by Intersil Company is an Integrated Circuit (IC) which is applied on the checking and protection for 3 series or 4 series Smart Battery [9] (shown in Fig.4). It combines the advantages of similar products made in TI and Microchip Company. The characteristics of X3100 are as follows:

(1)It has the functions as protecting over voltage, lack of voltage and over flow automatically;

(2)The delay time and release time of protection can be set;

(3)It has the circuit of inner voltage regulation; (4)It has balance control interfaces, and can open several interfaces synchronously;

(5)the protection parameters can be set by SPI port;(6)It has the mechanism of short circuit protection and recovery check;

(7)Its inner current can enlarge circuit to improve the accuracy of measuring;

(8)It has the function of $\mathrm{OV}$ low-voltage charge control;

(9)It has the unique function of load checking;(10)it has many work models.

In Normal model, x3100 checks periodically the voltage of each battery to see that whether it has the protection state of over voltage and lack of voltage; meanwhile, it checks the current constantly to see whether it has the state of over flow; and the protection value of these states can be set by MCU. When one or several protection state appear, x3100 operates automatically to close CFET or DFET, and marks the happening events on their register; MCU can reads these events to get their actions; MCU can control FET, balance circuit and current measure gains by modifying the value of register. When the over discharge happens, x3100 automatically closes CFET and DFET, and closes the output of rectifier voltage to enter the model of sleep to avoid the discharge further.
In checking model, x3100 exports selected Battery voltage or Rsensor voltage in AO port. The output of AO port is voltage analog signals. MCU measures the voltage and current by its own A/D and algorithms.

\section{C. $M C U$}

MCU is the central part of the system. MCU controls all functions of the system: initializing x3100; controlling x3100 to measure voltage and current; temperature measuring; electric capacity calculation; charge ways control; the judge and realization of protection; balance management[10][11]; the calculation of sbdata parameters; SMBus communication, etc. Because of the inner strong resources of MCU, its peripheral circuit is very simple relatively, shown in Fig.5.

(1) Clock system

GC1318 has multi-clocks selection system, and it can use various clocks resource. Considering the cost, the external 32.768K sub-clock and internal 2M RC clock of MCU are adopted.

(2) Flash structure of program and online programming

The structure of program area is flash structure, and it can be updated many times. Even if Pack is encapsulated, it can also be updated online by communication interfaces. The whole areas can be divided into Boot region and application region; the application region can be operated from Boot region, but the Boot region can't be operated in application region; thus the loader programs are stored in Boot region; when needing to update, the loader programs can be run by suitable way to realize the data update of program of application region; Boot region has special structure and lock position, so it can only use special tool to write loader programs on it; the data in Boot region must not be operated when it is used.

Here, the loader programs are written on MCU; then the various application programs are developed according the function request of different application schemes; finally, they can be downloaded online. Some application 
modifications are also updated online. It is very convenient and can save resource.

(3) LED display and key-press

In the application of Pack, there are requirements for states and electric indication. In our scheme, 5 LED are used, and they can indicate electric capacity and some states according given ways.

The system has a key-press. When the key-press is pushed, it can implement LED indication. It also can be used to boot when updating online.

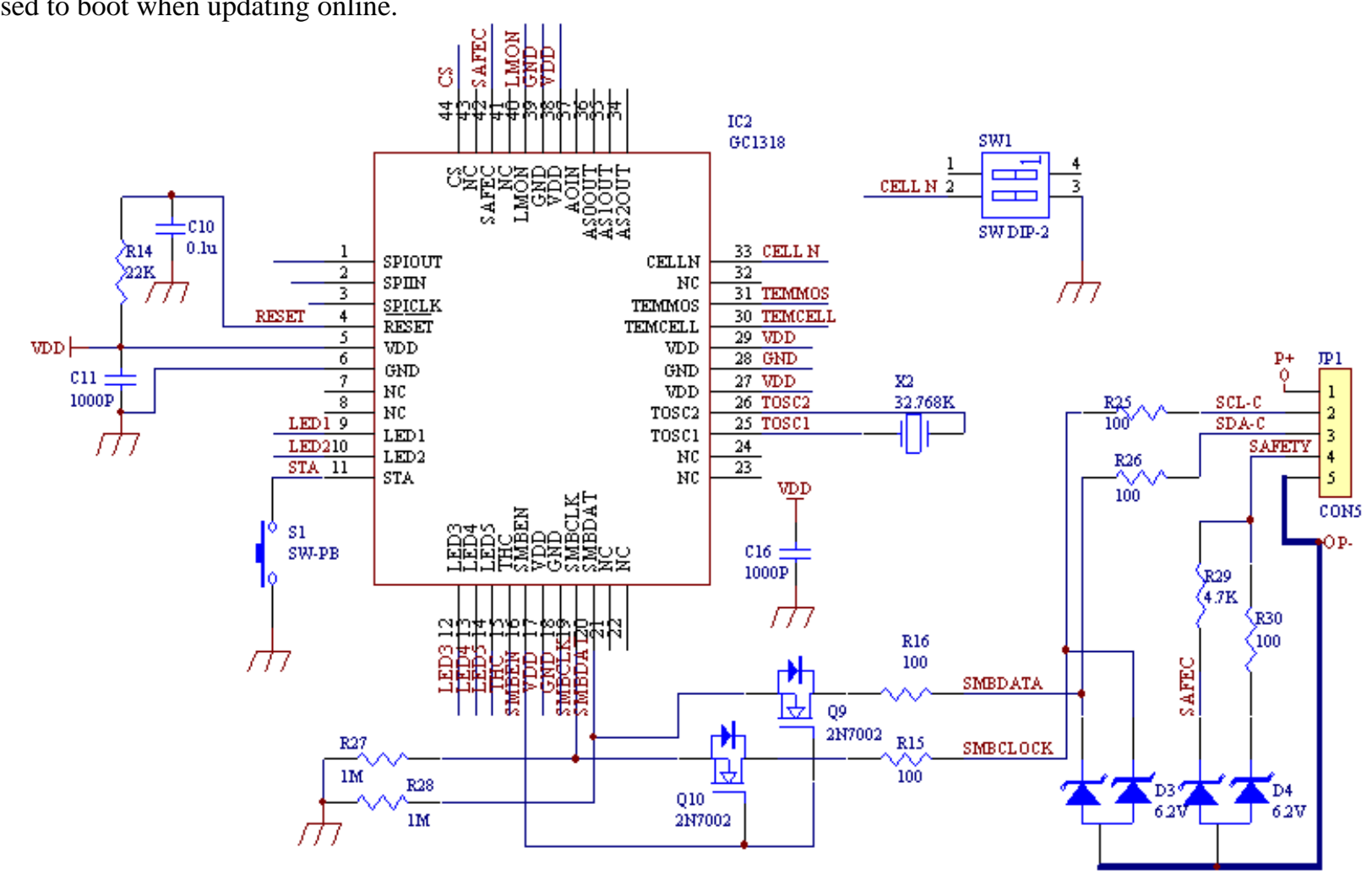

Fig.5 the relative circuit diagram of MCU

\section{The Software Design of Management System}

Software design adopted the way of structured programming. Making full use of the characteristics of $\mathrm{C}$ Compiler provided by developing tool, C Language Programming was adopted [12][13]. Software design should consider adequately the expandability and the transferability, and the bottom SMBus protocol and communication software can be made into general function, which can provide middleware by some way. Considering the special protocols of different laptop computers, it must provide the function of upgrade online for software; considering Intellectual Property Rights, it must provide the function of file encrypting and encrypting transport when upgrading online; so it must consider encrypting problem in software design.

System software mainly includes 7 models: system initialization model, parameters measuring model (includes the measuring of parameters as voltage, current, temperature, etc), data calculation and electric capacity management model, SMBus communication model,
(4)SMBus communication

SMBus is the standard bus structure of Smart Battery system components, and it is a kind of bus which is similar to IIC. SBS-IF defines perfect bus protocols included circuit structure, electric parameters, data structure and check way of transport format, etc. The latest version is SMBus 2.0. 
According to the sampling time, current measured is converted into electric capacity unit (mA); according to charge process or discharge process, the operation of addition and subtraction of electric capacity can be implemented to record current electric capacity of system; all parameters orders which include percent parameters and time parameters are calculated according to Smart Battery Data protocols; The parameters of Cycle of system operation need to be calculated; The calculation and storage of study are achieved when the system studies own FCC (full charge capacity).

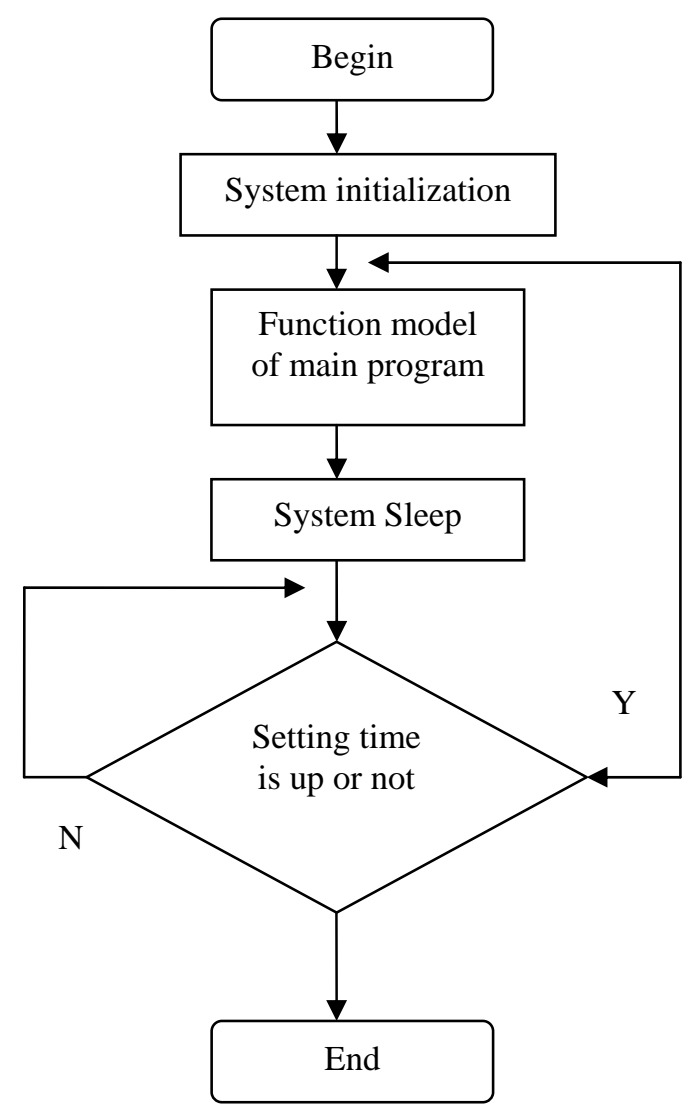

Fig.6 The main flow chart of management system software

(4) Communication model

Smart Battery system communicates with Host and Charger and exchanges order parameters by SMBus. The system controls the hardware of MCU and adopts interruption ways to finish the task of communication; the system supports the reading and writing of words data and block data; the system allows some parameters to be written into and reading all parameters; it will actively broadcast charge voltage, charge current and warning information when required.

(5) Sates processing model

The system will deal with its own states and judge and release the protection states of voltage, current and temperature according to the measuring and calculation data and the reading states of x3100. This model firstly uses queuing algorithm to mark the voltage of battery in order to compare conveniently. The order 0x16 in Sbdata is Battery Status, whose each position is a status indication, including charge/discharge indication, fullcharge indication, no-charge indication, temperature warning indication and over discharge indication, etc. This model also finishes that how to deal with these indications.
(6) Control processing model

Based on the measuring, calculating and processing of the former models, this model controls and operates the system, including Smart charge control, balance control, FET control and Smart electric capacity control, etc. Smart charge control is that software controls the conversion of pulse and constant current-constant voltage to realize the optimal charge way and charge result according to the charge speciality of Li-Ion. The software checks continuously the difference between batteries by balance algorithm; if satisfying the balance conditions, then it opens balance control to make the discrepant batteries reach balance by balance circuit of the hardware; thus it can keep the consistency of battery and increase the capacity of batteries and prolong their life. This system adopts sliding windows balance algorithm. This model controls the charge FET and discharge FET by communication with $\mathrm{x} 3100$.

(7) Time processing model

This model centrally deals with the calculation of various time used by the system, including he calculation of system self-discharge time, the calculation of various 
protection judge delay and the calculation of control time, etc.

The functions of the models above are independent, and communicate with each other by parameters, states signal and interruption. After the system being electrified, it begins to enter the work cycle of measure, calculation and management. Various function models, timer, interruption and watchdog all do their own job to make sure that the system has a reliable, safe and high effective work.

\section{CONCLUSIONS}

In this paper, the hardware and software design of Smart Li-Ion Battery management system based X3100 was introduced, and specific hardware design and software design schemes were elaborated. In the control of MCU - GC1318, the system realized the Smart management for battery and completed automatically some functions such as measure, calculate, communicate and protect. The system is characterized by little components, low cost, reliable operation, perfect function and comprehensive protection. The system has been applied on several types of laptop computer such as IBM , SONY and it is in good working.

\section{ACKNOWLEDGMENT}

This paper is supported by the Natural Science Foundation of Shandong Province (No. Y2008G03), Scientific research project of Shandong Province(No. 2009GG10001017) and independent innovation program of Colleges and Universities of Jinan City(No.200906002.) The authors also would like to express appreciation to the anonymous reviewers for their very helpful comments on improving the paper.

\section{REFERENCE}

[1] LIANG Jing. The price advantage of Netbook is outstanding and its market increases fiercely. http://tech.sina.com.cn/it/2009-0430/18343055047.shtml,2009.4.30

[2] Development Research Centre of the State Council. The delivery quantity of the global Notebooks hopefully increases fiercely $30 \%$ this year.http://www.drcnet.com.cn/DRCNet.Common.Web/D ocViewSummary.aspx?docid=1824166\&Leafid=979, 208-02-28

[3] LI Donghong. The sale quantity of Notebook in Chinese market will break up 15,000,000 sets in 2009.http://it.sohu.com/20090319/n262894609.shtml,200 9.3.19.

[4] Lin Feng; WangYuezhong. Design and Realization of Management System of Smart Lithium Batteries . Control \& Automation, 2005,21(3):78-81.

[5] SBS-IF. Smart Battery System Manager Specification, Version 1.0(Release B). 1999-08-09

[6] Intel. System Management Bus (SMBus) Specification, Version 2.2003-08-03

[7] SBS-IF.Smart Battery Charger Specification, Version 1.1. 1998-12-15

[8] Duracell and Intel.Smart Battery Data Specification, Version 1.1.1998-12-15

[9] Intersil. X3100 Datasheet. http://www.intersil.com/products/deviceinfo.asp?pn=X31 00\#data, 2002.12

[10] CHEN Shou-ping, ZHANG Jun, FANG Ying-min, LIANG Yi. Peculiarity analysis and proportion management of power batteries . Chinese Battery Industry, 2003,8(6):268-270

[11] W.F.Bentley. Cell Balancing Considerations for Lithiumion Battery Systems. Battery Conference on Applications and Advances, 1997:223-226.

[12] HE Limin. The Advanced Course of Single Chip Micyoco-Application and Design. Beijing: Aeronautics and Astronautics University Press, 2000

[13] WANG Xingzhi, ZHAI Cheng,WANG Shan. The Antiinterference Technology Application System of Single Chip. Beijing: Aeronautics and Astronautics University Press, 2000.2 